\title{
High Frame Rate Vector Velocity Estimation using Plane Waves and Transverse
} Oscillation

Jensen, Jonas; Stuart, Matthias Bo; Jensen, Jørgen Arendt

Published in:

Proceedings of IEEE International Ultrasonics Symposium

Link to article, DOI:

10.1109/ULTSYM.2015.0423

Publication date:

2015

Document Version

Peer reviewed version

Link back to DTU Orbit

Citation (APA):

Jensen, J., Stuart, M. B., \& Jensen, J. A. (2015). High Frame Rate Vector Velocity Estimation using Plane Waves and Transverse Oscillation. In Proceedings of IEEE International Ultrasonics Symposium IEEE. https://doi.org/10.1109/ULTSYM.2015.0423

\section{General rights}

Copyright and moral rights for the publications made accessible in the public portal are retained by the authors and/or other copyright owners and it is a condition of accessing publications that users recognise and abide by the legal requirements associated with these rights.

- Users may download and print one copy of any publication from the public portal for the purpose of private study or research.

- You may not further distribute the material or use it for any profit-making activity or commercial gain

- You may freely distribute the URL identifying the publication in the public portal 


\title{
High Frame Rate Vector Velocity Estimation using Plane Waves and Transverse Oscillation
}

\author{
Jonas Jensen, Matthias Bo Stuart and Jørgen Arendt Jensen \\ Center for Fast Ultrasound Imaging, Dept. of Elec. Eng. Bldg. 349, \\ Technical University of Denmark, DK-2800 Lyngby, Denmark
}

\begin{abstract}
This paper presents a method for estimating 2-D vector velocities using plane waves and transverse oscillation. The approach uses emission of a low number of steered plane waves, which result in a high frame rate and continuous acquisition of data for the whole image. A transverse oscillating field is obtained by filtering the beamformed RF images in the Fourier domain using a Gaussian filter centered at a desired oscillation frequency. Performance of the method is quantified through measurements with the experimental scanner SARUS and the BK $2 \mathrm{L8}$ linear array transducer. Constant parabolic flow in a flow rig phantom is scanned at beam-to-flow angles of 90,75 , and $60^{\circ}$. The relative bias is between $\mathbf{- 1 . 4 \%}$ and $\mathbf{- 5 . 8} \%$ and the relative std. between $5 \%$ and $8.2 \%$ for the lateral velocity component at the measured beam-to-flow angles. The estimated flow angle is $\mathbf{7 3 . 4}^{\circ} \pm \mathbf{3 . 6}^{\circ}$ for the measurement at $75^{\circ}$. Measurement of pulsatile flow through a constricted vessel demonstrate the application of the method in a realistic flow environment with large spatial and temporal flow gradients.
\end{abstract}

\section{INTRODUCTION}

Ultrasound blood flow estimation is of diagnostic value for investigating hemodynamic problems in the human cardiovascular system. Conventional ultrasound imaging systems are limited to only estimate the axial flow velocities along the ultrasound beam and suffer from a very low frame rate. Several methods have been proposed to estimate the full 2-D velocity vector without the need for angle correction, e.g., speckle tracking [1], directional beamforming [2], multibeam methods [3], and transverse oscillation (TO) [4]. The TO method has been FDA approved for clinical use and employs focused beams in line-by-line imaging, however, it is limited by a frame rate, which can be too low for capturing complex flow phenomena and full flow dynamics.

Alternative imaging methods such as synthetic aperture imaging (SA) using either spherical or plane waves (PW) have been proposed to increase the frame rate and improve image quality. An image of the entire insonified region is created for each emission resulting in a low-resolution image. By using a few broad insonifications, the low-resolution images can be summed to form a high-resolution (HR) image. SA has been used for flow estimation [5], and plane waves for 2-D motion estimation [6] and blood flow imaging using speckle tracking [7]. While especially the latter method is computationally demanding, the TO approach is relatively inexpensive in terms of number of calculations.

The objective of this paper is to show the feasibility of TO implemented in a fast imaging sequence using multiple plane waves to obtain flow estimates for a large field of view. The proposed method uses steered plane wave emissions, which are combined to HR images. The TO fields are generated in the Fourier domain, and computational inexpensive autocorrelation estimators are used for blood vector velocity estimation. A similar approach was proposed in [8], [9], but used only a single plane wave and applied a phase-based block matching method for tissue motion estimation.

\section{MethodS}

This section describes the method for vector velocity estimation, which is based on the TO approach [4]. A lateral oscillation in the pulse-echo field is introduced along with the conventional axial oscillation, so that the received signals become sensitive to both an axial and lateral motion in the field. The TO field is usually created in the receive beamforming by changing the apodization function to contain two separated peaks. However, a lateral oscillation can also be generated in the Fourier domain, known as $k$-space, to provide better control over the lateral oscillation wavelength. This is accomplished by using a filter in the Fourier domain, which filters the beamformed image in the lateral dimension to only select $k$-space components around a desired lateral oscillation frequency.

The process is illustrated in Fig. 1. Beamformed data for a HR image has a $k$-space as illustrated in Fig. 1 (top). An oscillation in the axial direction is centered at the pulse center frequency, while there is no oscillation laterally. A filter $G\left(f_{z}, f_{x}\right)$ consisting of Gaussian windows centered around a desired TO frequency $f_{0 x}$,

$$
\begin{aligned}
G\left(f_{z}, f_{x}\right)=\exp \left(-2\left(\pi \sigma_{x}\right.\right. & \left.\left.\left(f_{x}-f_{0 x}\right)\right)^{2}\right) \\
& +\exp \left(-2\left(\pi \sigma_{x}\left(f_{x}+f_{0 x}\right)\right)^{2}\right),
\end{aligned}
$$

where $\sigma_{x}$ is the width of the Gaussian window and $f_{x}$ is the lateral oscillation frequency, is illustrated in Fig. 1 (middle). A multiplication of the filter and the Fourier transformed image gives the TO image in Fig. 1 (bottom). The image has been filtered in the lateral dimension, while the axial dimension is untouched. Any values of the parameters $f_{0 x}$ and $\sigma_{x}$ can in principle be chosen, but it should be ensured only to choose $k$-space components containing energy from flow and not only noise. By having an effective $F$ \# that is relatively small, energy is retained for larger $f_{x}$. 


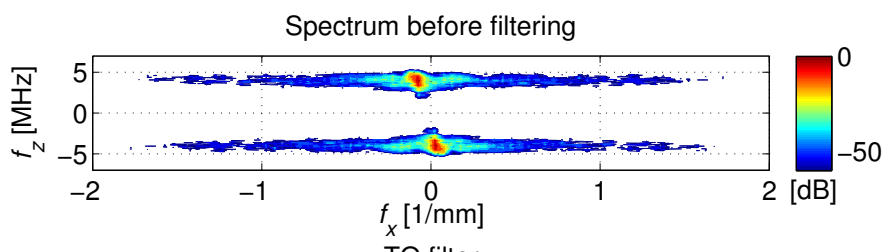

TO filter
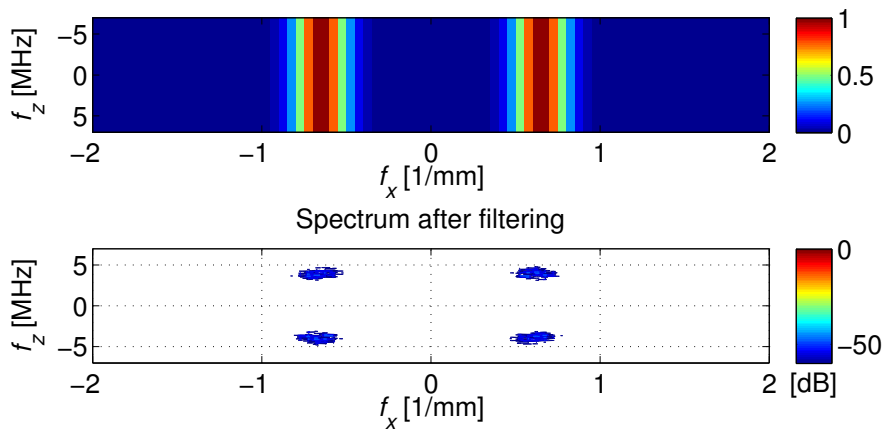

Fig. 1. The 2-D Fourier spectrum of a beamformed RF image is shown in the top figure. The spectrum of the TO filter $G\left(f_{z}, f_{x}\right)$ is shown in the middle, and the spectrum of beamformed RF image after applying the TO filter is shown in the bottom figure. The resulting RF image contains transverse oscillations with a mean lateral frequency according to the desired oscillation frequency of the TO filter.

The directional information of the flow is preserved by applying the Hilbert transform on the TO image (spatial domain) for each of the lines in the lateral direction. This avoids having a spatial quadrature between two beamformed signals.

The actual mean lateral oscillation frequency $\bar{f}_{x}$ at a given depth can be estimated from the TO image and used directly in the velocity estimator:

$$
\bar{f}_{x}=\frac{\int_{-f_{s z} / 2}^{+f_{s z} / 2} \int_{-f_{s x} / 2}^{+f_{s x} / 2} f_{x}\left|H\left(f_{z}, f_{x}\right)\right|^{2} d f_{z} d f_{x}}{\int_{-f_{s z} / 2}^{+f_{s z} / 2} \int_{-f_{s x} / 2}^{+f_{s x} / 2}\left|H\left(f_{z}, f_{x}\right)\right|^{2} d f_{z} d f_{x}},
$$

where $f_{s z}$ and $f_{s x}$ are axial and lateral sampling frequencies, respectively, and $H\left(f_{z}, f_{x}\right)$ is the Fourier transform of the TO image. Echo canceling is performed by subtracting the mean value across all the emissions from the RF signals. A standard fourth order autocorrelation estimator for the transverse velocity component is employed as proposed in [10] and with the estimated mean $\bar{f}_{x}$.

\section{EXPERIMENTAL SETUP}

A BK Ultrasound 2L8 linear array transducer with a center frequency of $4.1 \mathrm{MHz}$ and $0.55 \lambda$ pitch is connected to the experimental SARUS scanner [11]. A duplex imaging sequence is implemented, one for B-mode and one for flow. For the Bmode sequence, 33 plane wave emissions equally spaced from $-22^{\circ}$ to $22^{\circ}$ are used. For the flow sequence, plane waves are emitted at three angles $\left(-15^{\circ}, 0^{\circ}\right.$, and $\left.15^{\circ}\right)$ and a Tukey window is used in transmit to reduce the artifacts from edge waves. The system pulse repetition frequency $f_{p r f}$ is $7.5 \mathrm{kHz}$ giving an effective $f_{p r f, e f f}=f_{p r f} /(3+1)=1875 \mathrm{~Hz}$. Parabolic constant flow in a $6 \mathrm{~mm}$ radius tube in a flow rig system is scanned and the volume flow is measured by a magnetic flow meter
TABLE I

VARIED PARAMETERS

\begin{tabular}{lc}
\hline Variable parameter & Value \\
\hline Beam-to-flow angle & $60, \mathbf{7 5}, 90\left[^{\circ}\right]$ \\
Number of emitted PWs & $1, \mathbf{3}, 5,9$ \\
$f_{0 x}$ & $1,1.25, \mathbf{1 . 5 3}, 2,4[\mathrm{~mm}]$ \\
$\sigma_{x}$ & $0.5,1, \mathbf{1 . 5}, 2[\mathrm{~mm}]$ \\
Number of HR images & $5,10,22, \mathbf{4 5}, 90$ \\
\hline
\end{tabular}

Default conditions are shown in bold.
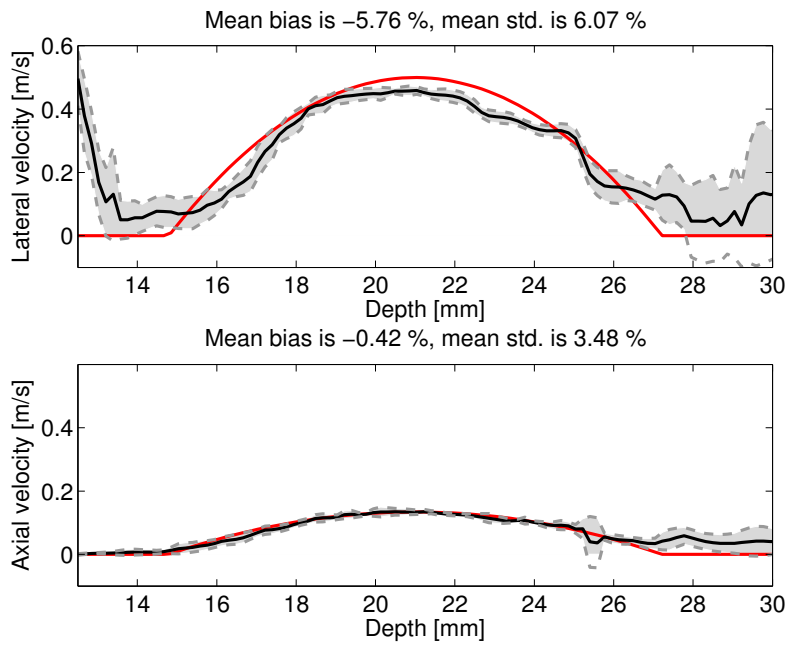

Fig. 2. Measured velocity profiles at a $75^{\circ}$ beam-to-flow angle. Lateral (top) and axial (bottom) velocity estimates with the true velocity profiles in red.

for reference (MAG 3000, Danfoss, Nordborg, Denmark). The vessel is at a depth of $22 \mathrm{~mm}$, and flow with a peak velocity of $0.5 \mathrm{~m} / \mathrm{s}$ is measured at beam-to-flow angles of 90,75 , and $60^{\circ}$. Table I lists the parameters that are varied in the study, and the parameters indicated with bold are for the default setup.

Pulsating flow was also scanned using an in-house fabricated flow phantom comprised of a straight tube with a concentric constriction of $36 \%$. The tube was surrounded by a tissue-mimicking material consisting of $15 \%$ polyvinyl alcohol cryogel, $1 \%$ silicon dioxide, $0.3 \%$ potassium sorbate, and $83.7 \%$ distilled water [12]. A blood-mimicking fluid was circulated through the tube in a closed loop circuit, and flow was generated by a CompuFlow 1000 system (Shelley Automation, Toronto, Canada).

Data were processed off-line, and delay-and-sum beamforming was performed using a dynamic apodization in receive with an $F \#=1$.

\section{RESULTS}

\section{A. Constant flow}

Velocity profiles for a measurement at a $75^{\circ}$ beam-to-flow angle using the default setup are shown in Fig. 2, where the top figure shows mean and standard deviation (std.) of the profile for the lateral velocity component. The relative bias for the lateral component is $-5.8 \%$. Selections of $45 \mathrm{HR}$ images are used for each velocity estimate and with an overlap of $75 \%$ between each selection, the frame rate is $167 \mathrm{~Hz}$. 


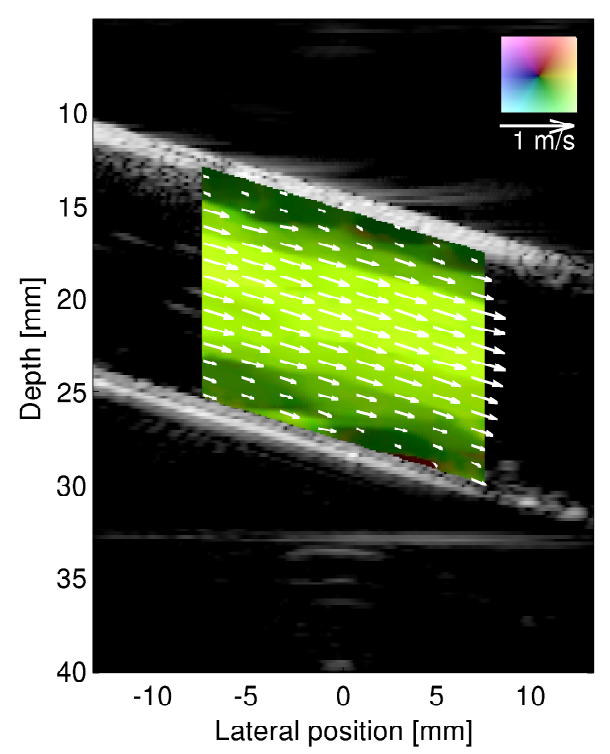

Fig. 3. Vector flow image of a measurement on the flow rig at a beam-to-flow angle of $75^{\circ}$. The flow is constant and parabolic with a peak velocity of 0.5 $\mathrm{m} / \mathrm{s}$.

A vector flow image for the measurement at $75^{\circ}$ is shown in Fig. 3, where the arrows indicate direction and magnitude of the flow. The beam-to-flow angle was estimated from the velocity data to be $73.4^{\circ} \pm 3.6^{\circ}$ at the center line.

Fig. 4 shows the performance of the lateral velocity component when four parameters have been varied. The relative bias is shown with a solid line, while the relative std. is a dashed line. Measurements at beam-to-flow angles of 60,75 , and $90^{\circ}$ are shown in Fig. 4(a), and the relative std. is lowest at $90^{\circ}$ $(5.6 \%)$, while it increases for smaller beam-to-flow angles.

The performance of the method, when changing the number of emitted plane waves are shown in Fig. 4(b). The experiment was performed at $75^{\circ}$ beam-to-flow angle, with a flow sequence of $9 \mathrm{PWs}$ emitted from $-15^{\circ}$ to $15^{\circ}$, and the peak velocity of the flow reduced to $0.25 \mathrm{~m} / \mathrm{s}$ to avoid aliasing. The number of PWs used for each HR image was then varied in the post-processing. The relative std. is constant and around $5 \%$ as a function of the number of emitted PWs, while the bias is smallest for 1 and for 9 PWs. The motivation for using steered PWs is that a larger aperture is synthesized, i.e., the lateral resolution is improved and more energy is collected for larger $f_{x}$. A larger number of emitted PWs reduces $f_{p r f, e f f}$ and the maximum detectable velocity, but should decrease the std., theoretically. It was, however, not the case with the current setup.

The result of varying parameters for the TO filter, i.e., the desired lateral oscillation frequency $f_{0 x}$ and width of the Gaussian window $\sigma_{x}$ is shown in Fig. 4(c) and (d), respectively. It is calculated for the default measurement setup at $75^{\circ}$. While the bias and std. are almost constant for a changing $\sigma_{x}$, they are more influenced by $f_{0 x}$. The std. increases from $5 \%$ to $12.5 \%$, when reducing $1 / f_{0 x}$ from $1.53 \mathrm{~mm}$ to $1 \mathrm{~mm}$. It indicates that (a)

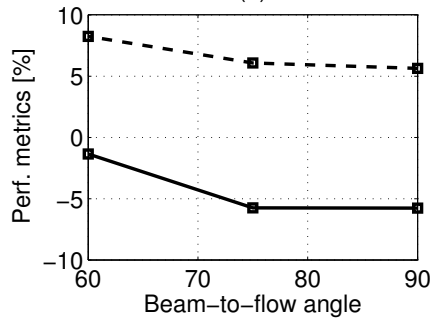

(c)

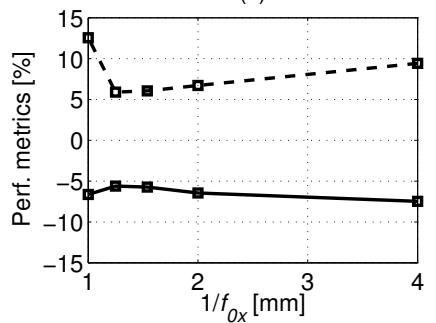

(b)

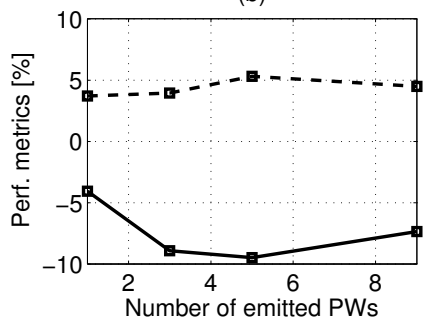

(d)

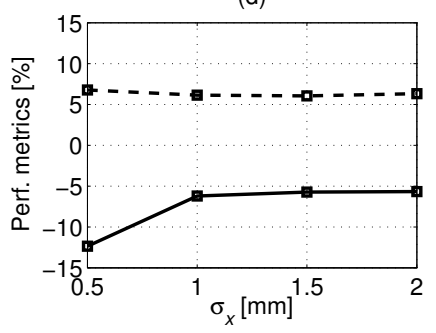

Fig. 4. Performance metrics for the lateral velocity component as a function of various parameter settings: Relative mean bias (solid line) and relative mean std. (dashed line).
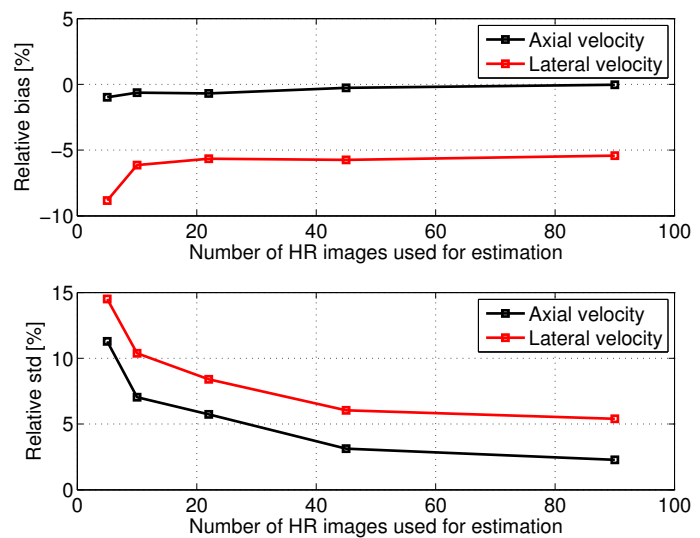

Fig. 5. Relative bias (top) and std (bottom) for a variation in the number of HR images used for velocity estimation. The beam-to-flow angle is $75^{\circ}$.

more energy is picked up from noise by the TO filter at $1 / f_{0 x}=$ $1 \mathrm{~mm}$. The smallest bias and std. are obtained by setting $1 / f_{0 x}$ $=1.53 \mathrm{~m}$ and $\sigma_{x}=1.5 \mathrm{~mm}$.

The relative bias and std. as a function of the number of HR images used for velocity estimation are shown in Fig. 5 for the axial and lateral velocity components. The beam-to-flow angle is $75^{\circ}$ and the bias is $-5 \%$ and std. reaches $5 \%$ for the lateral component, when using $90 \mathrm{HR}$ images, while the axial velocity is unbiased. The std. follows the expected $1 / \sqrt{N}$ trend, where $N$ is the number of HR images.

\section{B. Pulsatile flow}

A carotid flow profile is generated by the pump with a peak volume flow set to $3 \mathrm{~mL} / \mathrm{s}$ and a cardiac period of $0.84 \mathrm{sec}$. Three seconds of data were acquired and processed with the TO method. A vector flow image from peak systole is shown in Fig. 6 and from diastole in Fig. 7. The lateral velocity estimate $v_{x}$ in the center of the constricted tube is shown as a function of time in the bottom figures. Back-flow occur after 

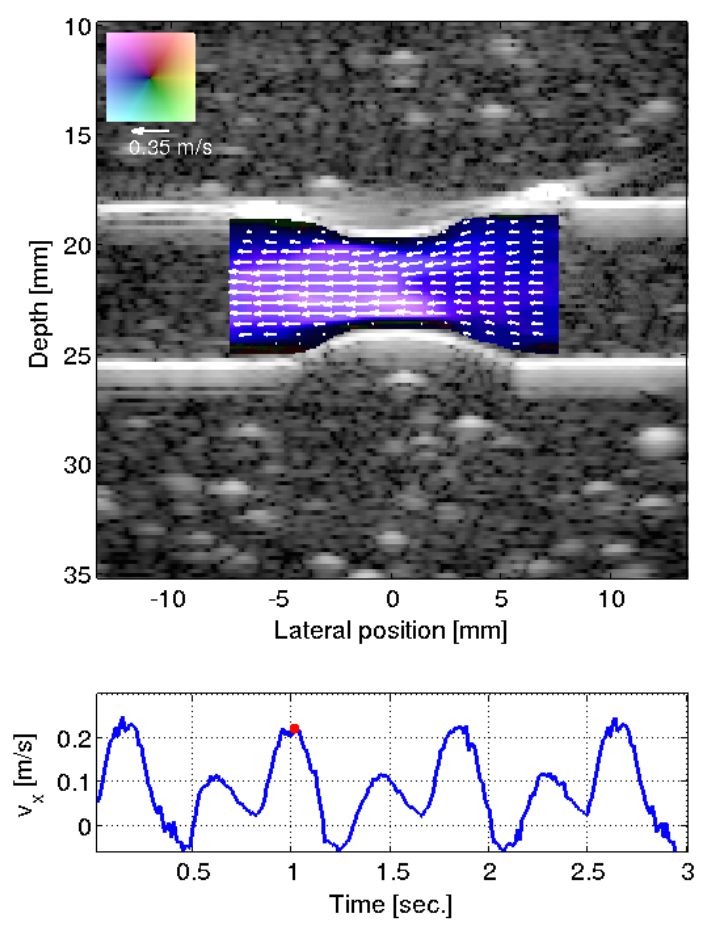

Fig. 6. Measurement of constricted vessel with pulsatile flow. The top figure shows the vector flow image at peak systole, and the bottom figure is the lateral velocity profile estimated in the center of the constriction.

the systole, which can be due to the vessel compliance. By dividing the profile of $v_{x}$ into three segments of length of a cardiac period, the mean std. of the profile is calculated to be $4.9 \%$ relative to the peak velocity.

\section{Discussion ANd CONCLUSION}

A high-frame rate method for estimating 2-D vector velocities using plane waves and transverse oscillation were presented. Three steered plane waves were emitted and the beamformed images were summed and used for flow estimation. A transverse oscillating field was obtained by filtering the beamformed RF images in the Fourier domain. The method provides large flexibility, since beamformation is performed once, and TO is generated subsequently at a desired wavelength and window width, which adaptively can be changed depending on the type of flow. The bias and std. were measured to be $-5.8 \%$ and $5.6 \%$, respectively, for fully transverse flow. The frame rate of the method was $167 \mathrm{~Hz}$ and can be changed depending on the number of HR images and overlap of selections used for estimation.

\section{ACKNOWLEDGEMENT}

This work was supported by grant 82-2012-4 from the Danish National Advanced Technology Foundation and by BK Ultrasound.

\section{REFERENCES}

[1] G. E. Trahey, J. W. Allison, and O. T. von Ramm, "Angle independent ultrasonic detection of blood flow," IEEE Trans. Biomed. Eng., vol. BME-34, pp. 965-967, 1987.
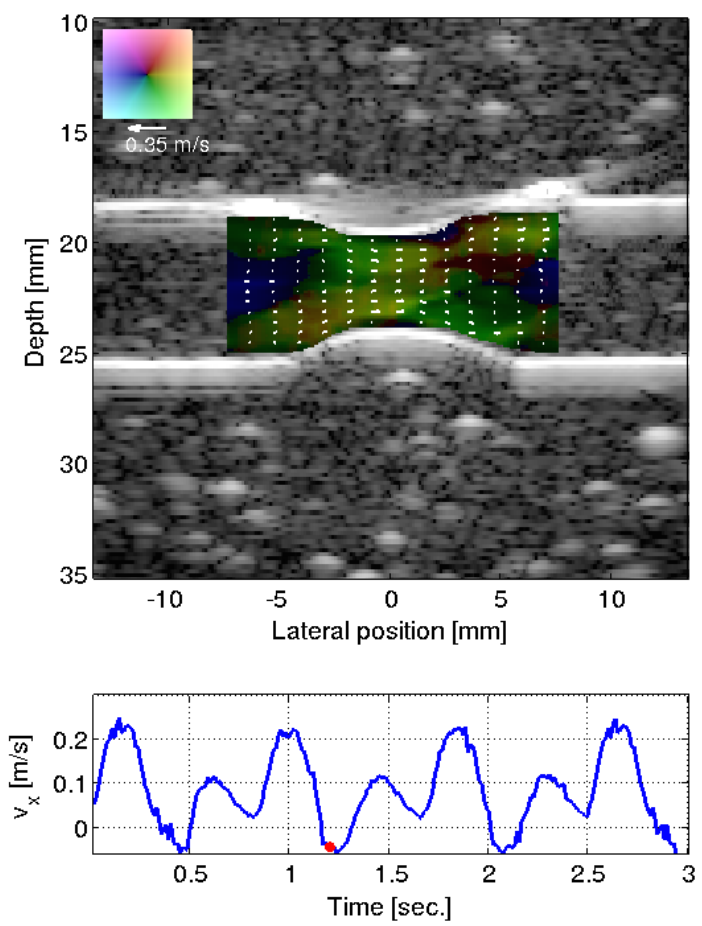

Fig. 7. Measurement of constricted vessel with pulsatile flow. The top figure shows the vector flow image at diastole, and the bottom figure is the lateral velocity profile estimated in the center of the constriction.

[2] J. A. Jensen, "Directional velocity estimation using focusing along the flow direction: I: Theory and simulation," IEEE Trans. Ultrason., Ferroelec., Freq. Contr., vol. 50, pp. 857-872, 2003.

[3] B. Dunmire, K. W. Beach, K.-H. Labs., M. Plett, and D. E. Strandness, "Cross-beam vector Doppler ultrasound for angle independent velocity measurements," Ultrasound Med. Biol., vol. 26, pp. 1213-1235, 2000.

[4] J. A. Jensen and P. Munk, "A new method for estimation of velocity vectors," IEEE Trans. Ultrason., Ferroelec., Freq. Contr., vol. 45, pp. $837-851,1998$.

[5] S. I. Nikolov and J. A. Jensen, "In-vivo Synthetic Aperture Flow Imaging in Medical Ultrasound," IEEE Trans. Ultrason., Ferroelec., Freq. Contr., vol. 50, no. 7, pp. 848-856, 2003.

[6] M. Tanter, J. Bercoff, L. Sandrin, and M. Fink, "Ultrafast compound imaging for 2-D motion vector estimation: application to transient elastography," IEEE Trans. Ultrason., Ferroelec., Freq. Contr., vol. 49, pp. 1363-1374, 2002

[7] J. Udesen, F. Gran, K. L. Hansen, J. A. Jensen, C. Thomsen, and M. B. Nielsen, "High frame-rate blood vector velocity imaging using plane waves: Simulations and preliminary experiments," IEEE Trans. Ultrason., Ferroelec., Freq. Contr., vol. 55, no. 8, pp. 1729-1743, 2008.

[8] S. Salles, A. J. Y. Chee, D. Garcia, A. C. H. Yu, D. Vray, and H. Liebgott, "2-D arterial wall motion imaging using ultrafast ultrasound and transverse oscillations," IEEE Trans. Ultrason., Ferroelec., Freq. Contr., vol. 62, no. 6, pp. 1047-1058, 2015.

[9] F. Varray and H. Liebgott, "An alternative method to classical beamforming for transverse oscillation images: Application to elastography," IEEE 10th Int. Symp. Biomedical Imaging, pp. 716-719, 2013.

[10] J. A. Jensen, "A new estimator for vector velocity estimation," IEEE Trans. Ultrason., Ferroelec., Freq. Contr., vol. 48, no. 4, pp. 886-894, 2001.

[11] J. A. Jensen, H. Holten-Lund, R. T. Nilsson, M. Hansen, U. D. Larsen, R. P. Domsten, B. G. Tomov, M. B. Stuart, S. I. Nikolov, M. J. Pihl, Y. Du, J. H. Rasmussen, and M. F. Rasmussen, "SARUS: A synthetic aperture real-time ultrasound system," IEEE Trans. Ultrason., Ferroelec., Freq. Contr., vol. 60, no. 9, pp. 1838-1852, 2013.

[12] S. S. Lai, B. Y. S. Yiu, A. K. Poon, and A. C. Yu, "Design of anthropomorphic flow phantoms based on rapid prototyping of compliant vessel geometries," Ultrasound Med. Biol., vol. 39, no. 9, pp. 16541664, 2013. 